Archives

$6 \mid 1990$

Varia

\title{
Les forêts entre rêves et réalités
}

\section{François Sigaut}

\section{OpenEdition}

\section{Journals}

Édition électronique

URL : http://journals.openedition.org/ccrh/2861

DOI : $10.4000 /$ ccrh.2861

ISSN : 1760-7906

Éditeur

Centre de recherches historiques - EHESS

Édition imprimée

Date de publication : 15 octobre 1990

ISSN : 0990-9141

Référence électronique

François Sigaut, "Les forêts entre rêves et réalités ", Les Cahiers du Centre de Recherches Historiques [En ligne], 6 | 1990, mis en ligne le 20 mars 2009, consulté le 19 avril 2019. URL : http:// journals.openedition.org/ccrh/2861 ; DOI : 10.4000/ccrh.2861

Ce document a été généré automatiquement le 19 avril 2019

Article L.111-1 du Code de la propriété intellectuelle. 


\title{
Les forêts entre rêves et réalités
}

\author{
François Sigaut
}

1 Dans cette contribution, je voudrais présenter, ou plutôt illustrer, deux idées que je crois assez générales relativement à la forêt. La première, c'est que la notion de forêt est des plus variables d'une société, d'une époque à une autre; ce qui naturellement pose un problème aux études comparatives. La seconde, c'est que l'espace forestier au sens minimum du terme, c'est-à-dire entendu comme l'opposé de l'espace civilisé, est un des lieux où les tendances les plus lourdes d'une société se donnent libre cours.

2 Je n'insisterai pas sur cette seconde idée, parce que ce n'est encore pour moi qu'une impression vague. Elle m'a été inspirée par le livre de R. Hardy et N. Séguin, Forêt et société en Mauricie $^{1}$, et par la visite des musées de la région. Il est clair que dans ces immenses mines de bois qu'étaient les forêts canadiennes au XIXe siècle et jusqu'au milieu du Xxe, l'exploitation capitaliste s'est déployée à visage découvert, sans le moindre souci de ménager les ressources et les hommes. Aujourd'hui, une enquête sur les chantiers forestiers du Brésil ou de Bornéo montrerait probablement la même situation. C'est, logiquement en forêt que le capitalisme est le plus sauvage. Mais il n'est pas le seul. En France par exemple, c'est au XIX ${ }^{\mathrm{e}}$ siècle une espèce d'utopie administrative qui s'exprime dans l'espace forestier. «Avec ses gardes au statut paramilitaire, son corps d'ingénieurs recrutés au concours et formés dans une Grande école, avec ses traditions, son idéologie et son immense méfiance pour tout ce qui n'est pas elle, l'administration des Eaux-etForêts aura certainement été pendant près de deux siècles ce que nous avons eu de plus semblable à l'administration chinoise d'ancien régime ", ai-je écrit à propos de L'homme aux bois d'Andrée Corvol $^{2}$ et en songeant aussi au bel article de B. Kalaora et D. Poupardin sur ce sujet ${ }^{3}$.

3 La liste est longue des fantasmes dont l'espace forestier est ou a été le support réel ou fictif. Au Japon et peut-être aussi en Allemagne, la forêt est matrice de la société (A. Berque). Un peu partout en Eurasie, elle est refuge pour les bons et méchants. Ermites et renonçants vont y rechercher une solitude au moins symbolique, les brigands y guettent les voyageurs et les amoureux vont s'y ébattre à l'abri des regards indiscrets : Port-Royal-des-champs, la forêt de Bondy et les bois de Chaville illustrent ces trois images 
en région parisienne. Mais il y a encore la forêt espace de guerre. Je ne parle pas des révoltes paysannes d'autrefois ni des maquis de la dernière guerre. Je parle de cette " guerre du feu » qui se répète chaque été avec une monotonie dérisoire dans le Midi de la France. Car c'est bien d'une guerre qu'il s'agit. «Les avions en formation de combat pilonnaient le chantier. Le feu en déroute se repliait sur lui-même, la cadence des bombardements ne lui laissait pas le temps de souffler. Les hommes en bleu franchissaient la ligne de défense aérienne pour porter des coups à l'ennemi... $»^{4}$. Près de deux cents pages de cette encre, recoupant d'autres témoignages, et venant en contrepoint à l'évidente inadéquation des méthodes, ne laissent guère de place au doute. Le but n'est pas de prévenir et de limiter les dégâts du feu de la façon la plus rationnelle possible, comme, par exemple, les forestiers américains s'y efforcent avec intelligence ${ }^{5}$. Le but est, pour ceux qu'on appelle à juste titre, les « soldats du feu », de s'approprier un terrain d'exercices où ils puissent réaliser le même genre d'exploits et éprouver le même genre d'émotions que leurs devanciers dans les guerres coloniales. Si on comprend cela, alors la récurrence des feux chaque été, la pauvreté des politiques de prévention, et même l'absurdité patente de certains moyens (les hydravions, par exemple, qui ne peuvent pas décoller quand le vent est fort, c'est-à-dire quand les risques de propagation sont les plus grands), n'ont plus rien de mystérieux. La pyromanie est une sorte de manie professionnelle chez certains pompiers, mais ce n'est pas de cela qu'il s'agit.

4 La guerre du feu sert maintenant trop d'intérêts pour que des méthodes plus pacifiques puissent encore être prises au sérieux. Dans ce climat guerrier, soigneusement entretenu avec l'aide des media, toutes les ressources sont naturellement monopolisées par l'armement. Même lorsque les armes s'évèrent peu efficaces, car il est toujours possible d'arguer qu'il en faut davantage. Au fond, et malgré les déplorations rituelles, les soldats $\mathrm{du}$ feu ne sont guère fâchés que les incendies reprennent chaque année, puisqu'ils sont leur raison d'être. La forêt des forestiers de l'École de Nancy était un fantasme de technocrates, une administration enfin débarrassée d'administrés. La forêt des soldats du feu est un rêve de militaires, une guerre sans ennemis. (Il y a bien des traîtres, qui sont les incendiaires réels ou supposés, mais c'est une autre histoire...)

J'en viens maintenant à la pluralité de la notion de forêt. On la constate dans le vocabulaire, qui, nulle part ailleurs peut-être, n'offre de mots désignant vraiment ce que nos géographes et nos botanistes entendent aujourd'hui par «forêt». Le mot forêt luimême, du bas-latin forestis, "extérieur au terroir ", n'avait à l'origine qu'un sens juridique qu'il a encore dans certains toponymes anglais (New Forest), et qui n'impliquait rien de précis quant à la végétation de l'endroit ainsi désigné. A l'inverse, les termes plus descriptifs quant à l'aspect du paysage paraissent tous combiner, dans des proportions variables mais de façon presque inséparable, les notions de «lieu boisé », de "terrain montueux ou élevé », et de " désert, lieu inhabité ». Tel était, semble-t-il, le cas du latin silva. Tel est aujourd'hui le cas des termes espagnols montado, monte (Cf. desmontar, "défricher »). En allemand, Wald et Berg signifient respectivement "forêt» et "montagne ", si on s'en tient aux dictionnaires. Mais dans les dialectes et la toponymie, les deux termes sont pratiquement interchangeables. La Forêt Noire (Schwarzwald) est un massif montagneux, et les Hauberge sont des parcelles de taillis essartées tous les vingt à vingt-cinq ans pour une ou deux récoltes de céréales. La toponymie anglaise offre un exemple de divergence remarquable : le Weald du Kent était une région forestière restée à peu près inhabitée jusqu'au début du XVII ${ }^{e}$ siècle ${ }^{6}$, alors que les Wolds du Gloucestershire sont des collines absolument dénudées servant de pâturages à moutons (les deux termes 
ont la même étymologie). En français, un mot comme bocage a donné lieu à de graves malentendus, dans la mesure où on a tiré argument de sa présence, dans tel ou tel texte médiéval, en faveur de l'existence à l'époque d'une forme de paysage identique au «bocage » de nos géographes actuels. Or, rien n'autorise une telle conclusion. Un bocage, c'était autrefois, un lieu boisé, rien de plus. Voici, par exemple, la définition qu'en donne le premier tome de L'Architecture dans L'Encyclopédie méthodique ${ }^{7}$ :

On peut définir le bocage comme une agrégation d'arbres plantés à dessein d'y faire trouver l'ombre et le frais, joints aux agréments de la nature embellie par les soins de l'homme. Le bocage tient le milieu entre le bois et le bosquet (voyez ces mots) : moins étendu et moins champêtre que l'un, il n'a pas les beautés de forme et d'ornemens que l'art et le goût savent donner à l'autre. ...Le bocage est fait pour les emplacemens découverts et même un peu élevés; il est destiné à servir de retraite pendant les ardeurs du soleil; ses ombrages pourtant ne doivent être ni épais ni sombres; il est bon que la lumière s'y joue à travers les rameaux: son caractère propre enfin est d'être riant.

6 On peut toujours objecter que ce sens architectural ou horticole de bocage n'a rien à voir avec celui qu'il a dans les dialectes et dans la toponymie qui en dérive. C'est vrai, mais tel est aussi exactement le cas du bocage des géographes. Qu'ils aient emprunté ce terme à la toponymie ou au vocabulaire de l'art de paysager, ce que j'ignore, ils lui ont donné un contenu beaucoup plus précis et en tous cas différent de celui qu'il avait avant eux.

7 Je termine cette mise en garde sur le vocabulaire en évoquant la jungle des romans d'aventures de notre enfance. J'en avais conservé l'idée qu'il s'agissait d'une forêt, et même de quelque chose d'assez proche d'une forêt vierge. Or, voici la définition qu'en donne M. Defauconpret, traducteur de Walter Scott, dans les Chroniques de la Canongate ${ }^{8}$ :

On appelle jungle, des terrains souvent marécageux, couverts de joncs, de roseaux, de glaïeuls, d'épines et de broussailles de toute espèce, qui s'élèvent à une hauteur assez considérable pour cacher les animaux féroces qui y établissent leur repaire.

8 Vérification faite, la jungle est bien encore, pour le Petit Robert, une « forme de savane couverte de hautes herbes, de broussailles et d'arbres, où vivent les grands fauves ». Mais cette définition me semble devoir davantage aux dictionnaires anciens dont s'est inspiré le rédacteur de l'article qu'à l'usage actuel. Le problème est que ce mot nous est aujourd'hui devenu trop familier pour que nous songions à aller en vérifier le sens dans le dictionnaire lorsque nous le rencontrons dans tel ou tel texte, et que, par conséquent, nous risquons de mettre de la forêt là où il n'y en avait pas.

9 L'exemple de la jungle est une bonne transition pour passer à la notion de la forêt comme parc de chasse. Voici d'abord un récit tiré de ces nombreux Gazetteers (répertoires géographiques) que les Anglais ont publié sur l'Inde au siècle dernier :

[...] les Ameers du Sinde (sic) se caractérisent par une passion extraordinaire pour les sports de plein air. Ils y sacrifient le bien-être et même l'existence de leurs sujets, lorsqu'ils vident pour les enclore de vastes terroirs cultivés dont ils font leurs shikargahs ou hunting-jungles. Il a quelques années, un des ces Ameers a dépeuplé, près de sa capitale, une grande étendue de terrain fertile et l'a convertie en shikargah, bien que cet acte de tyrannie insensé et monstrueux lui fit perdre un revenu annuel de 20. à 30.000 livres. Un autre fit raser un village au prétexte que les bruits de la population et des animaux domestiques dérangeaient le gibier d'une réserve voisine".

10 En réalité, le parc de chasse est quelque chose que l'on rencontre dans toute l'Eurasie, partout du moins où la population est assez dense et la société assez hiérarchisée. Les forests de l'Angleterre médiévale étaient, sinon des parcs, au moins des territoires de 
chasse où les activités économiques n'étaient aurorisées que dans les limites des lois protégeant le gibier. Toutes ces chasses n'étaient pas royales. Cranborne Chase, hérité par le général Pitt Rivers en 1880, avait été jusqu'en 1830 une de ces « chasses » médiévales, où les lois de protection du gibier avaient assuré involontairement la sauvegarde de nombreux sites archéologiques pré-médiévaux ${ }^{10}$; elle s'étendait sur 300.000 hectares. C'est souvent par la littérature naturaliste que nous sommes informés sur l'existence des parcs de chasse. En Chine, par exemple, au moins une espèce animale et une espèce végétale, le cerf du père David (elaphurus davidianus) et le Ginkyo biloba de nos jardins publics, doivent leur célébrité au fait qu'on ne les a pas découverts à l'état sauvage, mais dans des parcs impériaux. Et la Chine n'est pas seule dans ce cas. Le bison d'Europe n'a survécu jusqu'à nos jours que dans la forêt impériale de Bialowieza, à la frontière russopolonaise, où il fut d'ailleurs réintroduit après la dernière guerre à partir d'individus préservés dans des zoos. En dehors des élevages de taureaux de combat en Espagne, seuls quelques parcs anglais ont encore des boeufs considérés comme « sauvages ». Il y aurait encore de nombreux autres exemples. On peut évidemment s'interroger sur le caractère « sauvage » de cette sauvagerie artificiellement entretenue : un paradoxe qui, à mon sens, rend peu utilisables deux notions symétriques de sauvagerie et de domestication ${ }^{11}$. C'est peut-être en Allemagne, comme l'a montré Bertrand Hell dans un très beau livre ${ }^{12}$, que la reproduction de la sauvagerie est la plus minutieusement organisée.

11 Depuis le début de ce siècle, les naturalistes tendent à prendre le relais des chasseurs, et les gouvernements celui des rois et des grands seigneurs. Mais à cela près, la politique actuelle des parcs et réserves naturelles ne fait que prolonger une très ancienne tradition. Une tradition qui remonte au moins aux Achéménides, dont les parcs de chasse (avestique paraidaeza) sont devenus, par l'intermédiaire du grec et du latin écclésiastiques, le paradis de notre imaginaire actuel. L'étymologie du mot est claire : un paradis, c'est un enclos, c'est-à-dire un parc. Ce qui signifie, bien sûr, qu'il n'y de paradis qu'artificiels, et défendus par certains hommes contre d'autres hommes. A quelques milliers d'années d'intervalle, c'est la même triste histoire qui est arrivée aux paysans du Sind et à Adam et Eve. La différence, c'est que ceux-ci ont cru devoir diviniser celui qui les avait chassés. Nous n'avons pas fini de nous demander pourquoi.

Matériellement, l'aspect des forêts de chasse devait dépendre du type de chasse qu'on y pratiquait. J'ignore si la question a été traitée, mais elle me semble importante. Nos forêts de la région parisienne ont été aménagées surtout pour la chasse à courre, celles d'Europe centrale surtout pour la chasse au fusil ${ }^{13}$; il reste à préciser la nature exacte des différences qui en résultent. En Angleterre également, une promenade dans les bois donne quelquefois l'impression de différences difficiles à préciser et dont on ne sait si elles tiennent à des différentes méthodes d'exploitation du bois ou d'aménagement pour la chasse...

13 Après la chasse, il faudrait évoquer la forêt comme lieu de cueillette. Sujet immense, auquel Pierre Desffontaines avait consacré un chapitre, mais qui en mériterait bien davantage. Le problème est que nous voyons trop souvent la cueillette comme une activité marginale relevant de la pauvreté, de l'archaïsme ou du folklore, alors qu'elle atteignait souvent une importance quasi-industrielle. Nous en avons vaguement une idée en ce qui concerne les pays tropicaux, à cause par exemple de l'histoire du caoutchouc. Mais cela est beaucoup plus ancien. En Inde, par exemple, la plupart des tribus dites de chasseurs-cueilleurs par les ethnologues, vivent ou du moins vivaient de la vente d'un ou deux produits de cueillette spécifiques. En Europe et en France, il suffit de mentionner le 
hêtre (pour ses faînes oléagineuses, objet d'un commerce actif, par exemple à VillersCotterets), le tilleul (des villages entiers vivaient de la fabrication de cordes en écorce de tilleul), le chêne kermès ou garrus (l'arbre des garriques du midi sur lequel on récoltait la cochenille ou vermillion des teinturiers) ${ }^{14}$ ou le chêne dit à glands doux (Quercus Ilex, dont les fruits, aussi estimés que les châtaignes, étaient vendus, par exemple à Murcie et en Sardaigne) pour donner une idée de l'importance de la question. Les «forêts » qui produisaient toutes ces choses et bien d'autres, ne ressemblaient certainement pas à des forêts « ordinaires » : l'intensité des activités de cueillette qui s'y déroulaient devait très certainement leur donner une physionomie particulière.

Une cueillette très largement répandue était celle des feuilles d'arbre comme fourrage. Je ne reviendrai pas sur ce sujet dont $\mathrm{j}^{\prime}$ ai déjà traité ailleurs ${ }^{15}$, mais je rappelle que du Népal à la Finlande et à l'Espagne, la récolte des feuilles et l'aménagement des forêts pour cette récolte, ont affecté d'immenses étendues. En Europe centrale, c'est seulement avec le développement progressif de la faux à foin que cette activité est passée au second plan. Les forêts à feuilles du Népal prennent, du fait du dépouillement des arbres, un aspect pour nous tout à fait étrange. De tels paysages n'étaient pas inconnus chez nous; une photographie prise dans le Quercy par P. Desffontaines en donne une assez bonne idée ${ }^{16}$.

15 Je terminerai en rappelant que la forêt peut aussi être aménagée pour la production de céréales, pour l'agriculture. Là encore, je me borne à renvoyer à des travaux antérieurs ${ }^{17}$. Dernière remarque, enfin, il me semble qu'on a trop sous-estimé le caractère d'aménagement, et non seulement d'exploitation de la forêt qu'implique l'agriculture forestière. Le terme de "forêt secondaire », qu'on emploie généralement pour désigner une forêt qui a déjà été cultivée, est peut-être en partie responsable de cette sousestimation, dans la mesure où il n'implique pas l'idée d'aménagement intentionnel, lequel était pourtant souvent présent. En Europe, pratiquement toutes les forêts éssartées étaient des taillis à coupe réglée (par exemple les Hauberge des massifs montagneux rhénans). En Asie même, de tels systèmes étaient peut-être moins rares, et surtout plus anciens qu'il n'y paraît. On connaît le système dit des taungya, dont l'invention est traditionnellement attribuée à Sir Dietrich Brandeis, chef du Service forestier colonial de Birmanie en 1856. Or, ce système est attesté dans certaines régions de Chine du Sud depuis le début du XVII ${ }^{\mathrm{e}}$ siècle, et peut-être depuis le XII ${ }^{\mathrm{e}}$ siècle $^{18}$. Dans ce domaine où les recherches ont toujours été sporadiques, on ne peut exclure que cette découverte en appelle d'autres.

16 Faut-il conclure ? Ce sera pour dire que de l'extrême Ouest à l'extrême Est de l'Eurasie, la forêt est depuis longtemps un fait social à part entière, et que c'est seulement ainsi qu'on parviendra à la comprendre.

\section{Addendum}

Bien qu'ils soient rapportés dans la presse comme s'ils étaient une catastrophe, les incendies de forêts de ce mois d'août dans le Midi de la France ne constituent qu'une répétition banale d'événements qui surviennent régulièrement tous les cinq ans ou tous les dix ans. Les commentaires, eux, restent identiques à eux-mêmes, et, si on les compare avec ceux de la presse américaine au sujet des derniers incendies du Parc national de Yosemite $^{19}$, ils sont d'une pauvreté affligeante. En France, l'absence du point de vue scientifique est particulièrement remarquable. Un timide changement est peut-être toutefois en train de s'esquisser, si on en juge par deux articles parus dernièrement dans 
le journal Libération : «Le Pays d'Oc fouille sa mémoire végétale » et « Feu : la guerre des anciens contre les modernes $»^{20}$. Ces articles nous confirment,

$1^{\circ}$ que loin de régresser, la forêt méditérranéenne s'étend rapidement au contraire ; elle gagnerait par exemple $1 \%$ du territoire provençal chaque année ;

$2^{\circ}$ que cet envahissement indiscriminé par la forêt est un facteur qui accroît la fréquence et la gravité des incendies ;

$3^{\circ}$ qu'il coûte beaucoup moins cher de "lutter» contre les feux que de revenir à un aménagement du paysage qui les prévienne ;

$4^{\circ}$ que cette « lutte » contre les feux est devenue l'apanage d'une véritable corporation de militaires-fonctionnaires qui met un soin jaloux à en exclure les populations. L'enjeu est évident. Les méthodes lourdes prônées par cette corporation font certes, chaque année, la preuve qu'elles ne sont pas supérieures aux méthodes traditionnelles, mais elles ont deux avantages décisifs: elles entretiennent un flux important de crédits au profit de la corporation, et elles sont le moyen le plus facile et le plus spectaculaire d'atteindre un consensus social. Les élus locaux, en particulier, peuvent toujours réclamer davantage de canadairs, ça ne leur coûte pas grand chose et ça peut leur rapporter gros.

Tout cela, d'ailleurs, est connu. La seule question est de savoir quand ces faits émergeront dans un débat véritablement public, et non plus confidentiel.

\section{NOTES}

1. René HARDY et Normand SEGUIN, Forêt et société en Mauricie, 1830-1930, Montréal, Boréal Express et Musée National de l'Homme, 1984.

2. Andrée CORVOL, L'Homme aux bois, Paris, Fayard, 1987.

3. Bernard KALAORA et Denis POUPARDIN. «L'administration forestière entre 1860 et 1940. L'esprit de corps à travers les nécrologies ». Ethnologie française, 18, 1988, I. pp. 27-41.

4. Mireille DURAND, Le Feu sacré, Aix-en-Provence, Edisud, 1988. Passage cité dans Silva, 1989, 10, p. 21.

5. Stephen J. PYNE, Fire in America. A Cultural History of Woodland and Rural Fire, Princeton University Press, 1982 ; William ROMME et Don G. DESPAIN, « The Yellowstones fires ", Scientific American, 1989, 261. 5, pp. 21-29.

6. G.E. FUSSEL, «Quatre siècles de systèmes de culture dans le Kent, 1500-1900 », Études rurales, 1975, 59, pp. 7-33.

7. QUATREMERE de QUINCY, Encyclopédie méthodique. Architecture, tome 1, Paris, Panckoucke et Liège, Plomteux, 1788.

8. Walter SCOTT. Les Chroniques de la Canongate, Paris, Furne, 1830, trad. par M. Defauconpret, p. 450.

9. Edward THORNTON, A Gazetteer of the countries adjacent to India on the Northwest ; including Sinde, Afghanistan, Beloochistan, the Punjab and the neighbouring states, London, W.H. Allen \& Co., 1844.

10. François SIGAUT, « De la technologie à l'évolutionnisme, l'œuvre de Pitt Rivers (1827-1900) », Gradhiva, 8, 1990,.

11. François SIGAUT, « Critique de la notion de domestication », L'Homme, 1988, 108, pp. 59-71.

12. Bertrand HELL, Entre chien et loup, Paris, Éditions de la MSH, 1985. 
13. Pierre DESFFONTAINES. L'Homme et la forêt, Paris, Gallimard, 1933, pp. 66-70.

14. Dominique CARDON, Les Vers du rouge, Paris, Soc. française d'histoire des sciences et des techniques et Belin, 1990.

15. François SIGAUT, "Gli alberi da foraggio in Europa: significato tecnico ed economico ", Quaderni storici, 1982, 49, pp. 49-58 (Boschi : storia e archeologia); «L'arbre fourrager en Europe : rôle et évolution des techniques", in V. ALLEZARD et B. HUBERT (sous la direction de), La Forêt et l'élevage en région méditerranéenne française. Numéro hors série de la revue Fourrages, 1987, pp. 45-54.

16. Pierre DESFFONTAINES, op. cit., Pl. V.

17. François SIGAUT, L'agriculture et le feu. Paris-La Haye, Mouton, 1975 ; «Dynamique des systèmes culturaux traditionnels en Amérique tropicale ", Actes du XLII ${ }^{e}$ Congrès international des Américanistes, 1976, II, pp. 397-407 : "Swidden cultivation in Europe : a question for tropical anthropologists ", Information sur les sciences sociales. 1979, 18, 4/5, pp. 679-694.

18. Nicholas MENZIES, «Three hundred years of Taungya : a sustainable system of forestry in South China ». Human Ecology, 1988, 16. 4, pp.361-376; Michel CARTIER, communication personnelle, 1989.

19. Newsweek, 27 août 1990, pp. 44-45.

20. Libération, 11-12 août 1990, pp. 28-29 et 25-26 août 1990, pp. 19-20.

\section{AUTEUR}

\section{FRANÇOIS SIGAUT}

François SIGAUT est maître de conférences au Centre de Recherches Historiques. 